\title{
Email-based promotion of self-help for subthreshold depression: Mood Memos randomised controlled trial
}

\author{
Amy J. Morgan, Anthony F. Jorm and Andrew J. Mackinnon
}

\section{Background}

Subthreshold depression is common, impairs functioning and increases the risk of major depression. Improving self-help coping strategies could help subthreshold depression and prevent major depression.

\author{
Aims \\ To test the effectiveness of an automated email-based \\ campaign promoting self-help behaviours.
}

\section{Method}

A randomised controlled trial was conducted through the website: www.moodmemos.com. Participants received automated emails twice weekly for 6 weeks containing advice about self-help strategies. Emails containing general information about depression served as a control. The principal outcome was depression symptom level on the nine-item Patient Health Questionnaire (PHQ-9) (trial registration: ACTRN12609000925246)

\section{Results}

The study recruited 1326 adults with subthreshold depression. There was a small significant difference in depression symptoms at post-intervention, favouring the active group ( $d=0.17,95 \% \mathrm{Cl} 0.01-0.34)$. There was a lower, although non-significant, risk of major depression in the active group (number needed to treat (NNT) 25, 95\% Cl 11 to $\infty$ to NNT(harm) 57).

\section{Conclusions}

Emails promoting self-help strategies were beneficial. Internet delivery of self-help messages affords a low-cost, easily disseminated and highly automated approach for indicated prevention of depression.

\section{Declaration of interest}

A. J. Morgan is the author and developer of the Mood Memos website but derives no personal or financial benefit from its operation.
Depressive symptoms are thought to exist on a continuum, and the term 'subthreshold depression' has been used to describe symptoms of depression that are clinically relevant yet fall short of a diagnosis of major depressive disorder. Subthreshold depression poses a significant burden on the individuals affected as well as the wider community. ${ }^{1,2}$ Antidepressants have limited effectiveness for subthreshold depression ${ }^{3}$ and, although psychotherapies can be effective, ${ }^{4}$ it is not practical to disseminate them on a large scale because of workforce and healthcare resource limitations. A possible alternative approach to reducing the population burden of subthreshold depression is improving the usage of effective self-help coping strategies for depression symptoms. Members of the public often prefer self-help strategies to professional interventions, and self-help strategies are frequently adopted for mild levels of depression. ${ }^{5}$ However, many individuals with depression choose self-help strategies that are ineffective or even harmful (for example taking pain relievers or drinking alcohol) and may be unaware of more effective alternatives. ${ }^{6}$ Dissemination of information about effective self-help for subthreshold depression warrants investigation. ${ }^{7}$ Email has significant advantages over other communication channels, as it can reach a large number of targeted individuals using minimal resources. Therefore, this study evaluated the effectiveness of a series of automated emails based on persuasive communication techniques promoting effective self-help behaviours for subthreshold depression in a randomised controlled trial.

\section{Method}

\section{Study population and recruitment}

Full details of the study method have been described in the trial protocol. ${ }^{8}$ Recruitment to the study was from internet-based sources, including paid advertising with Google, Yahoo! and Facebook, posts on internet forums, promotion in email newsletters and links from other websites. Paid advertising with Google was directed at people who had searched for a short questionnaire online to find out whether they had depression. Other online advertising promoted the study to individuals with mild depressive symptoms as a way of receiving expert information or coping advice about depression via email. Participants joined the study by visiting the website www.moodmemos.com. The Mood Memos website contains clear links to The University of Melbourne and Orygen Youth Health as supporters of the study. Visitors to the Mood Memos website were screened for subthreshold depression with the nine-item Patient Health Questionnaire depression scale (PHQ-9). ${ }^{9}$ Results were categorised as subthreshold depression, an approximation of major depression or no depression. Subthreshold symptoms were defined as two to four symptoms of depression ${ }^{10}$ experienced more than half the days or nearly every day for 2 or more weeks, which have affected work, home or social functioning. Five or more symptoms of depression that had an impact on functioning were categorised as major depression. The no depression category was defined as not falling into the major depression or subthreshold depression categories, i.e. by experiencing fewer than two symptoms or not rating symptoms as having an impact on functioning. Participants were eligible for the trial if they were aged 18 years or over, had subthreshold symptoms of depression (as defined above), were not receiving treatment for depression from a health professional (but maintenance antidepressant medication for 6 months or more was allowed), were a resident of Australia, New Zealand, UK, Ireland, Canada or the USA, and had access to the internet at least weekly. Maintenance medication was allowed so that participants 
who had experienced a prior episode and were at risk of relapse could take part.

\section{Procedure}

The trial was registered at the Australian New Zealand Clinical Trials Registry (ACTRN12609000925246) and was approved by the University of Melbourne Human Research Ethics Committee (HREC 0931313). Individuals who screened positive for subthreshold depression were invited to participate in the study, were assessed for further inclusion criteria and were asked to submit a name and email address. The hyperlink to the pre-intervention assessment was sent to this email address. Once the pre-intervention assessment was completed, participants were randomised to the active group or control group. Immediately following randomisation, participants were sent their first Mood Memos email.

\section{Interventions}

The administration of the intervention was fully automated using PHP 5 and a MySQL 5 database. Participants were sent a Mood Memos email twice a week for 6 weeks by the automated system and had no interaction with a therapist. The active group received emails based on self-help strategies endorsed as effective and feasible by depression experts. ${ }^{11}$ The control group received emails containing general information about depressive disorders (see Appendix for an overview of the content of each Mood Memos email). The design of the active Mood Memos was a crucial component of the study, as merely providing information is not sufficient to change behaviour. The emails employed a variety of techniques to encourage and persuade the recipient to engage in the self-help behaviours. ${ }^{8}$ These included persuasive framing, tailoring, goal setting and limiting cognitive load. The emails included an explanation of each strategy, why it would work, tips on how it could be implemented, suggested solutions for implementation barriers, an appeal to commit to implementing the strategy and a reminder about previous strategies. The strategies were ordered in terms of their feasibility to carry out, with the most feasible strategy sent first. Feasibility rankings were taken from the original expert consensus study. ${ }^{11}$ The control group Mood Memos did not direct any action and were designed not to have any therapeutic value. Their role was to control for the effect of receiving emails with depression-related content.

\section{Outcomes}

Assessment points were at baseline, midway through the intervention (3 weeks post-baseline) and at the end of the intervention (6 weeks post-baseline). All assessment was self-rated and undertaken on the Mood Memos website. Participants received up to one reminder email to complete each assessment. The primary outcome was depression symptom severity at postintervention, assessed with the PHQ-9.9 ${ }^{9}$ This outcome was also categorised to yield probable depression diagnoses. The PHQ-9 is a well-validated and widely used measure of depression that assesses the frequency over the previous 2 weeks of the nine criterion-A symptoms of a DSM-IV major depressive episode. ${ }^{10}$ The PHQ-9 can be used either as a diagnostic algorithm to make a probable diagnosis of major depressive disorder or as a continuous measure of severity. Scores range from 0 to 27 and cut-points of 5, 10, 15 and 20 represent mild, moderate, moderately severe and severe levels of depression. ${ }^{12}$ Secondary outcomes were more general psychological distress, assessed with the Kessler Psychological Distress Scale (K10), ${ }^{13}$ and level of functioning, assessed with the Work and Social Adjustment Scale
(WSAS). ${ }^{14}$ The K10 measures 10 symptoms of mental health in the anxiety-depression spectrum. Scores range from 10 (no distress) to 50 (severe distress) using the Australian scoring method. ${ }^{15}$ It has good internal consistency ${ }^{16,17}$ and is a good discriminator of community 'cases' and 'non-cases' of DSM-IV disorders. ${ }^{18}$ The WSAS measures impairment in work, home management, social activities, private leisure activities and ability to form and maintain relationships. Scores range from 0 to 40 , with a score above 20 suggesting moderate to severe psychopathology, scores between 10 and 20 suggest significant functional impairment but less severe symptomatology, and scores below 10 are associated with subclinical populations. The scale has strong psychometric properties, including good internal consistency and good convergence with patient ratings of perceived improvement. ${ }^{14}$

\section{Sample size}

The size of the effect of the intervention was anticipated to be small, given that meta-analysis of previous trials of indicated prevention of depression showed a small effect size of $d=0.23$. $^{19}$ Similarly, unsupported internet-based treatment of depression has been shown to have a small effect size of $d=0.25,{ }^{20}$ consistent with the findings from a meta-analysis published more recently. ${ }^{21}$ A power analysis indicated that a sample of 393 per condition would give $80 \%$ power to detect a small effect size ( 0.2 standard deviations between conditions) on a continuous outcome measure assuming a correlation of 0.5 between pre- and post-intervention scores. ${ }^{22}$ Allowing for a potential drop-out rate of $33 \%,{ }^{23}$ the target total sample size was 1200 participants.

\section{Statistical analysis}

Analyses were undertaken on an intention-to-treat basis. Any participants who were randomised but withdrew from the study, or who took up alternative treatments, were included in the analysis as randomised. Primary and secondary outcomes were evaluated using mixed models for repeated measures. Relationships between observations at different measurement occasions were modelled as an unstructured covariance matrix. Degrees of freedom were estimated using Satterthwaite's approximation. Planned contrasts compared changes from preintervention to post-intervention between groups. Categorical outcomes were based on the PHQ-9 and were calculated with the same algorithm used during screening, i.e. the categories of subthreshold depression, probable major depression and no depression (or remission from subthreshold depression). Relative risk was calculated and tested for significance. The number needed to treat (NNT) to avoid one person developing major depression and to achieve one remission from subthreshold depression were calculated with $95 \%$ confidence intervals (CIs) using the method proposed by Bender. ${ }^{24}$ A negative confidence limit indicates that the CI for the NNT runs to infinity and includes the possibility that exposure to the intervention may reduce the likelihood of protection or remission. Thus the limit of the CI is the limit of the number needed to treat (harm) (NNTH; see $\operatorname{Altman}^{25}$ ).

Several potential predictors of attrition or depression score post-intervention were explored. These were pre-intervention PHQ-9 score; age; gender (female); history of depression (yes/ no); history of bipolar disorder or psychotic disorder (yes/no); mood disturbance (depressed mood or lack of interest/pleasure) at pre-intervention (yes/no); experience of one or more negative life events in the previous 6 months, as assessed by the List of Threatening Experiences (yes/no); ${ }^{26,27}$ confidence in their ability to help themselves with their depressive symptoms (coping selfefficacy), dichotomised to little or no confidence $v$. moderate or 
very confident; sought treatment from a health professional for their depressive symptoms during the study (yes/no); and highest level of education (primary/secondary school or trade $v$. bachelor or postgraduate degree). These predictors were entered into separate logistic regression analyses, with presence of data at post-intervention as the outcome variable. Separate linear regression analyses were run in the active group with postintervention PHQ-9 score as the outcome variable, adjusting for pre-intervention PHQ-9 score. Predictors that were significant at the $P<0.1$ level were retained for simultaneous entry in a hierarchical multiple regression analysis with pre-intervention score entered in the first block. Between-group effect sizes (Cohen's $d$ ) were calculated by dividing the difference between the two group means at post-intervention by their pooled standard deviation. We tested all effects at the $P<0.05$ level (two-tailed). Analyses were carried out using SPSS version 18 for Macintosh.

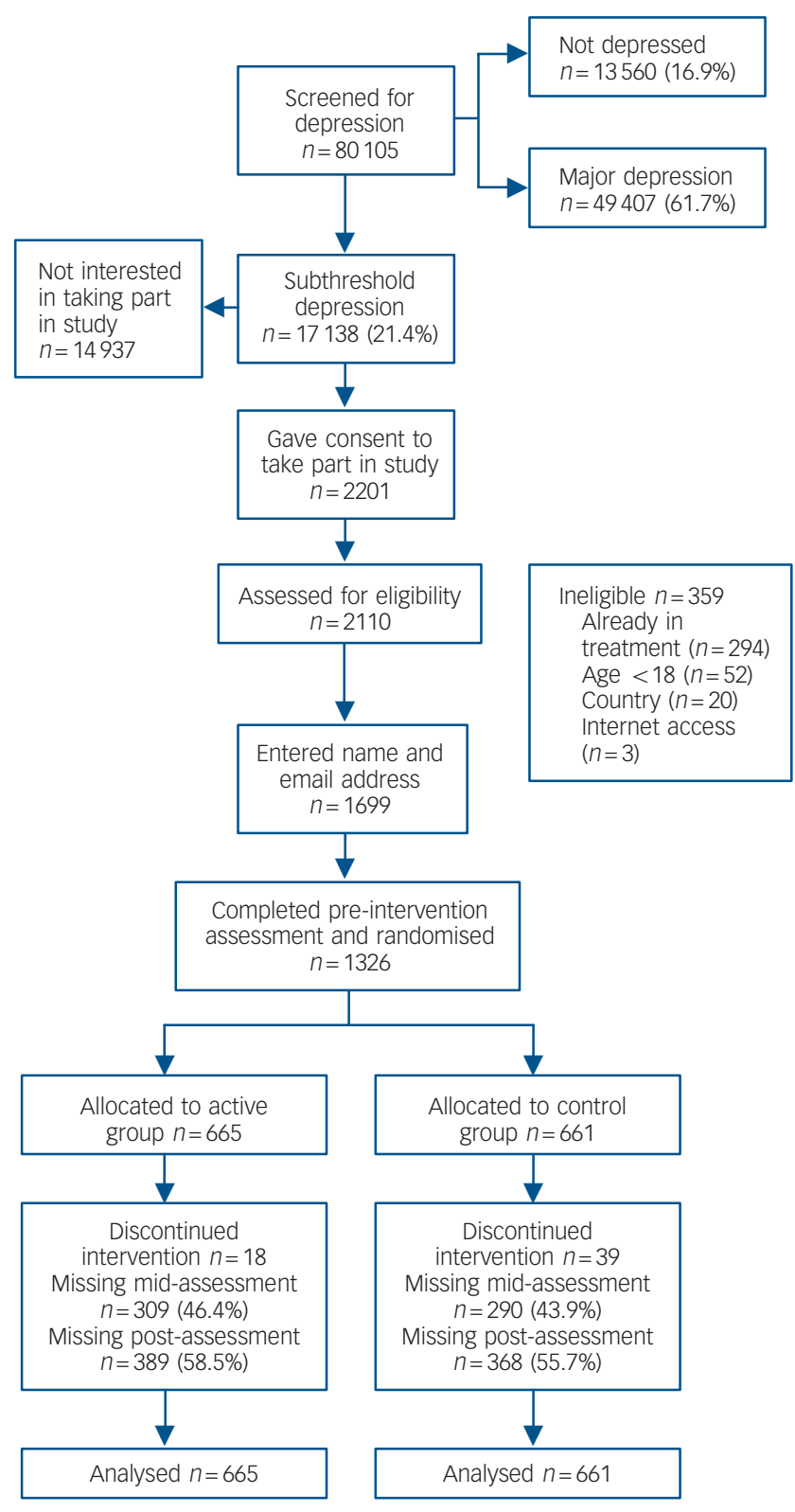

Fig. 1 Recruitment and retention of participants in the Mood Memos study.

\section{Results}

\section{Screening}

Figure 1 presents a flowchart of the recruitment and retention of participants in the trial. Recruitment took place between February 2010 and March 2011. More than 80000 PHQ-9 questionnaires were completed on the Mood Memos website. The majority of individuals scored in the major depression category, making them ineligible for participation. The final sample comprised 1326 participants who had subthreshold depression, gave consent to participate, met all eligibility criteria and completed the pre-intervention assessment.

\section{Participants}

Table 1 presents an overview of the sociodemographic characteristics of the sample. Participants ranged in age from 18 to 78 years, with a mean of 36 years. Over three-quarters of participants were female, nearly half the sample possessed at least a bachelor degree and nearly half reported a history of depression. As can be seen in the table, the two groups were well matched. At pre-intervention, there were no significant differences between the control and active groups on the PHQ-9, K10 and WSAS (Table 2). Reflecting inclusion criteria, scores on the PHQ-9 at pre-intervention were in the mild-to-moderate range $($ mean $=10.6$, s.d. $=1.97)$. Mood disturbance was present in $46 \%$ of the sample. Although participants did not qualify for a diagnosis of major depression, mean scores on the K10 suggested that they had high psychological distress ${ }^{28}$ and substantial functional impairment as indicated by mean scores on the WSAS.

\section{Attrition}

A small number of participants $(n=58,4.4 \%)$ elected to discontinue receiving the intervention. More participants in the control group withdrew from the Mood Memos emails $(5.9 \% v$. $\left.2.7 \%, \chi^{2}(1)=8.22, P=0.004\right)$. As shown in Fig.1, there was significant participant attrition. The attrition rate at the midintervention assessment was $45.2 \%$, which increased to $57.1 \%$ at the post-intervention assessment. The proportion of participants in each group who completed the post-intervention assessment was not significantly different $\left(\chi^{2}(1)=1.08, P=0.299\right)$. Completing assessments at post-intervention was significantly related to having a history of depression (odds ratio $(\mathrm{OR})=1.35,95 \% \mathrm{CI}$ $1.08-1.69, P=0.007)$, having a university education $(\mathrm{OR}=1.46$, $95 \%$ CI 1.17-1.81, $P=0.001)$ and lower depression scores at pre-intervention $\quad(\mathrm{OR}=0.90,95 \%$ CI $0.85-0.95, \quad P<0.001)$. Having complete data was not significantly related to age $(\mathrm{OR}=1.01, \quad 95 \% \quad$ CI $1.00-1.02, \quad P=0.113)$, being female $(\mathrm{OR}=1.21,95 \%$ CI $0.93-1.57, P=0.165)$, having a history of bipolar disorder or psychotic disorder $(\mathrm{OR}=1.49,95 \%$ CI $0.78-$ 2.84, $P=0.229)$, mood disturbance $(\mathrm{OR}=0.82,95 \%$ CI $0.66-1.03$, $P=0.082)$, coping self-efficacy $(\mathrm{OR}=1.07,95 \%$ CI $0.86-1.33$, $P=0.565)$ or experience of a negative life event in the previous 6 months $(\mathrm{OR}=0.79,95 \%$ CI $0.62-1.00, P=0.051)$.

\section{Outcome of the intervention}

Table 2 shows the means and standard deviations of the primary and secondary outcomes at each measurement occasion for each group. The overall interaction of time and group was significant for the PHQ-9 $(F(2,647.4)=3.10, P=0.046)$. Planned contrasts showed that the active group improved significantly more than the control group on the PHQ-9 between pre- and post-intervention $(t(627.4)=2.32, P=0.02)$. The mean difference in improvement was 


\begin{tabular}{|c|c|c|c|}
\hline Outcome & Active $(n=665)$ & Control $(n=661)$ & Total $(n=1326)$ \\
\hline Age, years: mean (s.d.) & $35.7(13.2)$ & $35.6(13.4)$ & 35.7 (13.3) \\
\hline Female, \% & 77.0 & 78.2 & 77.6 \\
\hline \multicolumn{4}{|l|}{ Highest education level, \% } \\
\hline Postgraduate degree & 20.3 & 17.5 & 18.9 \\
\hline Bachelor degree & 29.2 & 29.8 & 29.5 \\
\hline Trade-vocational diploma or certificate & 26.0 & 26.0 & 26.0 \\
\hline Secondary/high school or less & 23.9 & 25.9 & 24.9 \\
\hline Primary/elementary school or less & 0.6 & 0.8 & 0.7 \\
\hline \multicolumn{4}{|l|}{ Country of residence, \% } \\
\hline Australia & 52.6 & 46.1 & 49.4 \\
\hline UK & 28.3 & 32.8 & 30.5 \\
\hline Canada & 8.4 & 8.6 & 8.5 \\
\hline USA & 6.0 & 7.6 & 6.8 \\
\hline Ireland & 2.4 & 2.9 & 2.6 \\
\hline New Zealand & 2.3 & 2.0 & 2.1 \\
\hline History of depression, \% & 48.0 & 46.0 & 47.0 \\
\hline History of bipolar disorder or psychotic disorder, \% & 2.1 & 3.6 & 2.9 \\
\hline
\end{tabular}

\begin{tabular}{|c|c|c|c|c|}
\hline & \multicolumn{2}{|c|}{ Control } & \multicolumn{2}{|c|}{ Active } \\
\hline & Mean (s.d.) & $n$ & Mean (s.d.) & $n$ \\
\hline \multicolumn{5}{|c|}{ Patient Health Questionnaire-9 } \\
\hline Pre-intervention & $10.6(2.0)$ & 661 & $10.6(2.0)$ & 665 \\
\hline Mid-intervention & $9.1(4.5)$ & 371 & $8.5(4.4)$ & 356 \\
\hline Post-intervention & $8.3(5.0)$ & 293 & $7.5(4.7)$ & 276 \\
\hline \multicolumn{5}{|c|}{ Kessler Psychological Distress Scale-10 } \\
\hline Pre-intervention & $26.6(5.2)$ & 661 & $26.4(5.1)$ & 664 \\
\hline Mid-intervention & $23.0(6.3)$ & 371 & $22.0(6.2)$ & 356 \\
\hline Post-intervention & $21.9(7.2)$ & 292 & $20.3(6.6)$ & 276 \\
\hline \multicolumn{5}{|c|}{ Work and Social Adjustment Scale } \\
\hline Pre-intervention & $20.4(7.3)$ & 661 & $20.4(7.4)$ & 664 \\
\hline Mid-intervention & $17.8(8.2)$ & 371 & $17.2(8.6)$ & 356 \\
\hline Post-intervention & $15.9(9.3)$ & 292 & $14.8(9.3)$ & 276 \\
\hline
\end{tabular}

0.91 points (95\% CI $0.14-1.69)$. The effect size post-intervention was $d=0.17$ (95\% CI 0.01-0.34). Although the overall interaction between time and group for the K10 was not statistically significant $(F(2,655.9)=2.62, P=0.074)$, there was a significant difference between groups in change from pre-intervention to postintervention $(t(639.2)=2.22, P=0.027)$. The active group showed a mean improvement of 1.2 points (95\% CI $0.13-2.21$ ) more than the control group on the K10 scale $(d=0.22,95 \%$ CI $0.05-0.38)$. The interaction between time and group for the WSAS was not significant $(F(2,664.3)=1.35, P=0.261)$ and there were also no significant differences between groups on the change from pre- to post-intervention $(t(655.8)=1.63, P=0.104, d=0.12$, $95 \%$ CI -0.05 to 0.28$)$.

Categorical outcomes on the PHQ-9 are presented in Table 3. Among all participants at post-intervention, 15\% fell into the major depression category, $56 \%$ had achieved remission and $29 \%$ remained in the subthreshold depression category. The risk of developing major depression in the control group was not significantly higher than the active group, relative risk $(\mathrm{RR})=1.32(95 \%$ CI $0.89-1.98)$. The number needed to treat to prevent one case of major depression was 25 (95\% CI 11 to $\infty$ NNTH 57). The risk of remission from subthreshold depression in the control group was also not significantly lower than in the active group, $\mathrm{RR}=0.92$ (95\% CI 0.76-1.10). The number needed to treat to achieve one remission from subthreshold depression was 26 (95\% CI 8 to $\infty$ NNTH 23).

\section{Predictors of outcome}

Predictors of intervention outcome were analysed in the active group with multiple linear regression. Higher pre-intervention depression scores were a significant predictor of higher postintervention scores $(b=0.42$, s.e. $=0.15, P=0.005)$. Controlling for pre-intervention depression and other predictors, lower post-intervention depression scores were predicted by older age $(b=-0.06$, s.e. $=0.02, P=0.005)$ and no history of depression ( $b=1.58$, s.e. $=0.57, P=0.007)$. Higher coping self-efficacy was significantly associated with lower depression post-intervention when controlling for pre-intervention depression $(b=-1.19$, s.e. $=0.56, \quad P=0.036)$, but not when controlling for other predictors.

\section{Discussion}

\section{Main findings}

The automated Mood Memos emails were effective in reducing depression symptoms in adults with subthreshold depression. The use of emailed advice to change behaviour has been shown to be effective in improving diet and physical activity, ${ }^{29}$ but to our knowledge, this is the first study to show this approach also has potential for depression. Mean scores on the PHQ-9 in the group who received emails elaborating expert-endorsed self-help strategies declined from moderate to mild severity. ${ }^{9}$ Participants 
Table 3 Depression outcome per group at mid- and post-intervention

\begin{tabular}{|c|c|c|c|c|}
\hline \multirow[b]{2}{*}{ Outcome } & \multicolumn{2}{|c|}{ Mid-intervention } & \multicolumn{2}{|c|}{ Post-intervention } \\
\hline & $\begin{array}{l}\text { Control, } n(\%) \\
\quad(n=371)\end{array}$ & $\begin{array}{l}\text { Active, } n(\%) \\
\quad(n=356)\end{array}$ & $\begin{array}{l}\text { Control, } n(\%) \\
\quad(n=292)\end{array}$ & $\begin{array}{c}\text { Active, } n(\%) \\
\quad(n=276)\end{array}$ \\
\hline No depression & $157(42.3)$ & $178(50.0)$ & $158(54.1)$ & $160(58.0)$ \\
\hline Subthreshold depression & $153(41.2)$ & $120(33.7)$ & $85(29.1)$ & $81(29.3)$ \\
\hline Major depression & $61(16.4)$ & $58(16.3)$ & $49(16.8)$ & $35(12.7)$ \\
\hline
\end{tabular}

also showed a significant improvement in psychological distress, but psychological functioning did not improve significantly more than in the control group. Rates of major depression at the end of the study were lower in the active group, but the difference was not statistically significant. Similarly, remission from subthreshold depression was not significantly more frequent in the active group. Lower depression scores at post-intervention in the active group were predicted by older age, no history of depression and lower pre-intervention depression scores.

The effect size on the PHQ-9 $(d=0.17)$ was a similar magnitude to that found in other prevention of depression trials. ${ }^{19}$ Considering that these trials were generally face-to-face interventions delivered by mental health professionals, it is noteworthy that comparable results were achieved with automated emails. Effect sizes are generally smaller in preventive interventions than treatment studies, yet from a population perspective prevention is important because treatment can only avert a minority of the population burden from depression. ${ }^{30}$ The risk reduction in progression to major depression demonstrated in this study was also similar to that in other depression prevention studies. ${ }^{31}$ However, the sample sizes required to detect a significant reduction in incidence can be extremely large, ${ }^{32}$ and this study was not powered to detect such a reduction. The smaller effect on psychological functioning may have been because changes in functioning can take longer to occur than symptom changes. ${ }^{33}$ The 6-week study duration may have been too short to observe significant improvements in functioning.

Many individuals in the community prefer dealing with mental health problems on their $\mathrm{own}^{34}$ and believe self-help approaches can be effective. ${ }^{35}$ Although psychological treatments are moderately effective for subthreshold depression, ${ }^{4}$ they are not a viable solution to reducing the population burden of subthreshold depression. This is because healthcare resources are limited and many people do not seek professional treatment. There is a need for preventive interventions for depression that go beyond face-to-face interventions and can be widely disseminated. ${ }^{7}$ The Mood Memos trial promoted the usage of self-help strategies that are supported by evidence as being effective and that are analogous to health promotion campaigns used for other major sources of disease burden which have raised awareness about actions that can be taken to reduce risk of disease. Muñoz et $a l^{36}$ have also recognised the value of this approach, advocating for the use of preventive techniques that raise public awareness of how people can cope with subthreshold forms of depression. Although we found a small effect, this is balanced by the ease and low cost of disseminating the intervention to many people. Set-up costs of automated internet-based systems can be relatively high, but once developed, they have the advantage of scalability, in that more people can be reached at low incremental cost, compared with non-internet-based treatments. Further refinement of the email content, coupled with follow-up 'booster' emails or tailoring to recipient preferences, may enhance the effectiveness of this type of intervention.

\section{Strengths and limitations}

Attrition was substantial, which is common in internet-based interventions, particularly those with open recruitment. ${ }^{23,37}$ Attrition is a large problem in internet trials because participants are often less closely supervised and discontinuation can be achieved very easily. ${ }^{38}$ The reminder email to complete assessments led to an increase in completion of assessments, and an additional reminder email may have further reduced the level of attrition. The analysis techniques used retained all available data from participants yielding intention-to-treat estimates of effectiveness. However, these do make assumptions about missingness mechanisms. ${ }^{39}$ Confidence in these assumptions and the conclusions reached was enhanced by the inclusion of an additional occasion of measurement, midway through the intervention. This showed an emerging trend consistent with the post-intervention outcome. Reliance on self-report and the absence of a clinical interview to rule out physical causes for depressive symptoms may also be considered a limitation of the study. Inclusion of participants with physical causes of depression may have attenuated effects, as some participants' symptoms may not have been amenable to intervention with self-help.

The relatively low participation rate in individuals who screened positive for subthreshold depression may suggest there is low interest in this type of intervention in the community. Alternatively, it could have been caused by the recruitment method, where a large proportion of those screened visited the website via Google advertising to find out whether they had depression, rather than to necessarily seek help for their symptoms. Also, the study could not be promoted as a way of learning better ways of coping with depressive symptoms, as the control intervention had to remain credible. Issues around uptake of the intervention could be addressed in further evaluation under naturalistic conditions, rather than within the constraints of a randomised controlled trial.

As in other internet-based studies, there was a large proportion of well-educated participants. ${ }^{40}$ However, given that education was not a significant predictor of outcome, the intervention may well be suitable for those with a lower level of education. The majority of participants were female. This is not unexpected given that depression is more common in women, that women are more likely to seek help generally ${ }^{41}$ and that they are more likely to search for health information on the internet. ${ }^{42}$ It remains a challenge to reach individuals in the population who are less likely to seek help from health services for mental health problems, such as males with a low level of education.

A strength of the study was the active (placebo) control group design. Feedback from participants suggests that some found comfort from receiving the Mood Memos emails and felt less isolated. Yet the inclusion of a control group that also received emails means that the effect of the intervention was not solely due to these non-specific factors. The control group emails contained basic psychoeducation for depression, which can also 
be effective, ${ }^{43}$ so conclusions about the effectiveness of the intervention are stronger than if a wait-list or no-treatment control had been used.

\section{Implications}

Future research could investigate the effectiveness of the Mood Memos emails in people with clinical levels of depression, given its effectiveness for subthreshold depression and the gradient of response with level of symptomatology. For these people it would be appropriate to offer the intervention as an adjunct to professional treatment, rather than as a stand-alone treatment, due to its small effect and the absence of supervision.

Results from this randomised controlled trial show that a series of automated emails containing self-help advice is effective in reducing symptom levels in adults with subthreshold depression. Internet delivery of self-help messages is a scalable, easily disseminated intervention, that may help reduce the population disease burden of depression.

\section{Amy J. Morgan, BASC, BAppSci (Psychol)(Hons), MMM, Anthony F. Jorm, PhD, DSC, Andrew J. Mackinnon, PhD, Orygen Youth Health Research Centre, Centre for Youth Mental Health, University of Melbourne, Victoria, Australia}

Correspondence: Amy J. Morgan, Orygen Youth Health Research Centre, University of Melbourne, Locked Bag 10, Parkville, Victoria 3052, Australia Email: ajmorgan@unimelb.edu.au

First received 16 Aug 2011, final revision 24 Oct 2011, accepted 19 Dec 2011

\section{Funding}

Funded by a National Health and Medical Research Council Postgraduate Scholarship (567056) and a Sidney Myer Health Scholarship. Orygen Youth Health Research Centre receives funding from the Colonial Foundation.

\section{Acknowledgements}

Thanks to the working group of Sarah Bourchier, Laura Hart, Claire Kelly, Betty Kitchener and Siobhan Ryan. Thanks to David Jahshan for programming assistance with PHP and MySQL. Thanks to the websites and organisations that assisted with recruiting participants to the study.

\section{Appendix}

\section{Content overview of the Mood Memos emails}

\begin{tabular}{|c|c|}
\hline Order & Active \\
\hline 1 & Get out of the house. \\
\hline 2 & Fight the desire to do nothing. \\
\hline 3 & Set yourself a small goal and \\
\hline 4 & Eat well. \\
\hline 5 & Improve your sleep habits. \\
\hline 6 & $\begin{array}{l}\text { Do something you enjoy and } \\
\text { that gives you a sense of } \\
\text { achievement. }\end{array}$ \\
\hline 7 & $\begin{array}{l}\text { Talk to someone supportive } \\
\text { about your problems and how } \\
\text { you feel. }\end{array}$ \\
\hline 8 & Get active. \\
\hline 9 & $\begin{array}{l}\text { Do what has worked for you } \\
\text { in the past. }\end{array}$ \\
\hline 10 & $\begin{array}{l}\text { Let others know how you are } \\
\text { feeling. }\end{array}$ \\
\hline 11 & $\begin{array}{l}\text { Ask someone you trust to help } \\
\text { you get out and do things. }\end{array}$ \\
\hline 12 & Learn relaxation techniques. \\
\hline
\end{tabular}

Control

What is depression?

What types of depression are there?

Can depression cause physical

health problems? Depression and other mental health problems. What is the history of depression? What causes depression?

Who is at risk of depression?

How common is depression? Burden of depression.

Is depression a weakness?

Does depression re-occur?

Depression across cultures.

\section{References}

1 Backenstrass M, Frank A, Joest K, Hingmann S, Mundt C, Kronmüller K-T. A comparative study of nonspecific depressive symptoms and minor depression regarding functional impairment and associated characteristics in primary care. Compr Psychiatry 2006; 47: 35-41.

2 Cuijpers P, Smit F, Oostenbrink J, de Graaf R, ten Have M, Beekman A. Economic costs of minor depression: a population-based study. Acta Psychiatr Scand 2007; 115: 229-36.

3 Fournier JC, DeRubeis RJ, Hollon SD, Dimidjian S, Amsterdam JD, Shelton RC, et al. Antidepressant drug effects and depression severity: a patient-level meta-analysis. JAMA 2010; 303: 47-53.

4 Cuijpers $P$, Smit F, van Straten A. Psychological treatments of subthreshold depression: a meta-analytic review. Acta Psychiatr Scand 2007; 115: 434-41.

5 Jorm AF, Griffiths KM, Christensen H, Parslow RA, Rodgers B. Actions taken to cope with depression at different levels of severity: a community survey. Psychol Med 2004; 34: 293-9.

6 Jorm AF, Medway J, Christensen H, Korten AE, Jacomb PA, Rodgers B. Public beliefs about the helpfulness of interventions for depression: effects on actions taken when experiencing anxiety and depression symptoms. Aust N Z J Psychiatry 2000; 34: 619-26.

7 Jorm AF, Griffiths KM. Population promotion of informal self-help strategies for early intervention against depression and anxiety. Psychol Med 2006; 36 3-6.

8 Morgan AJ, Jorm AF, Mackinnon AJ. Protocol for a randomised controlled trial investigating self-help email messages for sub-threshold depression: the Mood Memos study. Trials 2011; 12: 11.

9 Kroenke K, Spitzer RL. The PHQ-9: a new depression diagnostic and severity measure. Psychiatr Ann 2002; 32: 509-15.

10 American Psychiatric Association. Diagnostic and Statistical Manual of Mental Disorders (4th edn) (DSM-IV). APA, 1994.

11 Morgan AJ, Jorm AF. Self-help strategies that are helpful for sub-threshold depression: a Delphi consensus study. J Affect Disord 2009; 115: 196-200.

12 Kroenke K, Spitzer RL, Williams JBW, Löwe B. The Patient Health Questionnaire somatic, anxiety, and depressive symptom scales: a systematic review. Gen Hosp Psychiatry 2010; 32: 345-59.

13 Kessler RC, Andrews G, Colpe LJ, Hiripi E, Mroczek DK, Normand SLT, et al. Short screening scales to monitor population prevalences and trends in non-specific psychological distress. Psychol Med 2002; 32: 959-76.

14 Mundt JC, Marks IM, Shear MK, Greist JM. The Work and Social Adjustment Scale: a simple measure of impairment in functioning. Br J Psychiatry 2002; 180: $461-4$.

15 Andrews G, Slade T. Interpreting scores on the Kessler Psychological Distress Scale (K10). Aust N Z J Public Health 2001; 25: 494-7.

16 Donker T, van Straten A, Marks I, Cuijpers P. Brief self-rated screening for depression on the Internet. J Affect Disord 2010; 122: 253-9.

17 Kessler RC, Barker PR, Colpe L, Epstein JF, Gfroerer JC, Hiripi E, et al. Screening for serious mental illness in the general population. Arch Gen Psychiatry 2003; 60: 184-9.

18 Furukawa TA, Kessler RC, Slade T, Andrews G. The performance of the K6 and $\mathrm{K} 10$ screening scales for psychological distress in the Australian National Survey of Mental Health and Well-Being. Psychol Med 2003; 33: 357-62.

19 Horowitz JL, Garber J. The prevention of depressive symptoms in children and adolescents: a meta-analytic review. J Consult Clin Psychol 2006; 74: 401-15.

20 Andersson G, Cuijpers P. Internet-based and other computerized psychological treatments for adult depression: a meta-analysis. Cogn Behav Ther 2009; 38: 196-205.

21 Cuijpers $\mathrm{P}$, Donker $\mathrm{T}$, Johansson R, Mohr DC, van Straten A, Andersson G. Self-guided psychological treatment for depressive symptoms: a metaanalysis. PLOS ONE 2011; 6: e21274.

22 Cohen J. A power primer. Psychol Bull 1992; 112: 155-9.

23 Christensen $\mathrm{H}$, Griffiths KM, Farrer L. Adherence in internet interventions for anxiety and depression. J Med Internet Res 2009; 11: e13.

24 Bender R. Calculating confidence intervals for the number needed to treat. Control Clin Trials 2001; 22: 102-10.

25 Altman DG. Confidence intervals for the number needed to treat. BMJ 1998; 317: 1309-12.

26 Brugha TS, Bebbington P, Tennant C, Hurry J. The List of Threatening Experiences: a subset of 12 life event categories with considerable long-term contextual threat. Psychol Med 1985; 15: 189-94. 
27 Brugha TS, Cragg D. The List of Threatening Experiences - the reliability and validity of a brief life events questionnaire. Acta Psychiatr Scand 1990; 82: 77-81.

28 Australian Bureau of Statistics. 4363.0.55.001 - National Health Survey: Users' Guide - Electronic, 2007-08. Australian Bureau of Statistics, 2009 (http://www.abs.gov.au/AUSSTATS/abs@.nsf/DetailsPage/ 4363.0.55.0012007-08)

29 Sternfeld B, Block C, Quesenberry Jr CP, Block TJ, Husson G, Norris JC, et al Improving diet and physical activity with ALIVE: a worksite randomized trial. Am J Prev Med 2009; 36: 475-83.

30 Andrews G, Issakidis C, Sanderson $\mathrm{K}$, Corry J, Lapsley H. Utilising survey data to inform public policy: comparison of the cost-effectiveness of treatment of ten mental disorders. Br J Psychiatry 2004; 184: 526-33.

31 Cuijpers $P$, van Straten A, Smit F, Mihalopoulos C, Beekman A. Preventing the onset of depressive disorders: a meta-analytic review of psychological interventions. Am J Psychiatry 2008; 165: 1272-80.

32 Cuijpers $P$. Examining the effects of prevention programs on the incidence of new cases of mental disorders: the lack of statistical power. Am J Psychiatry 2003; 160: 1385-91.

33 McKnight PE, Kashdan TB. The importance of functional impairment to mental health outcomes: a case for reassessing our goals in depression treatment research. Clin Psychol Rev 2009; 29: 243-59.

34 van Beljouw I, Verhaak $\mathrm{P}$, Prins $\mathrm{M}$, Cuijpers $\mathrm{P}$, Penninx B, Bensing J. Reasons and determinants for not receiving treatment for common mental disorders. Psychiatr Serv 2010; 61: 250-7.
35 Jorm AF, Nakane Y, Christensen H, Yoshioka K, Griffiths KM, Wata Y. Public beliefs about treatment and outcome of mental disorders: a comparison of Australia and Japan. BMC Med 2005; 3: 12.

36 Muñoz RF, Cuijpers P, Smit F, Barrera AZ, Leykin Y. Prevention of major depression. Annu Rev Clin Psychol 2010; 6: 181-212.

37 Melville KM, Casey LM, Kavanagh DJ. Dropout from internet-based treatment for psychological disorders. Br J Clin Psychol 2010; 49: 455-71.

38 Eysenbach G. The law of attrition. J Med Internet Res 2005; 7: e11.

39 Salim A, Mackinnon A, Christensen H, Griffiths K. Comparison of data analysis strategies for intent-to-treat analysis in pre-test-post-test designs with substantial dropout rates. Psychiatry Res 2008; 160: 335-45.

40 Titov N, Andrews G, Kemp A, Robinson E. Characteristics of adults with anxiety or depression treated at an internet clinic: comparison with a national survey and an outpatient clinic. PLoS One 2010; 5: e10885.

41 Carragher N, Adamson G, Bunting B, McCann S. Treatment-seeking behaviours for depression in the general population: results from the National Epidemiologic Survey on Alcohol and Related Conditions. J Affect Disord 2010; 121: 59-67.

42 Atkinson NL, Saperstein SL, Pleis J. Using the internet for health-related activities: findings from a national probability sample. J Med Internet Res 2009; 11: e4

43 Donker T, Griffiths K, Cuijpers $\mathrm{P}$, Christensen $\mathrm{H}$. Psychoeducation for depression, anxiety and psychological distress: a meta-analysis. BMC Med 2009; 7: 79 . 